\title{
The use of ultra-low-energy dynamic SIMS in the study of the tarnishing of silver
}

\author{
M.G. Dowsett ${ }^{\mathrm{a}, *}$, A. Adriaens ${ }^{\mathrm{b}}$, M. Soares ${ }^{\text {c }}$, H. Wouters ${ }^{\mathrm{d}}$, V.V.N. Palitsin ${ }^{\mathrm{a}}$, \\ R. Gibbons ${ }^{\text {a }}$, R.J.H. Morris ${ }^{\text {a }}$ \\ ${ }^{a}$ Advanced SIMS Projects, Department of Physics, Warwick University, Warwick, Coventry CV4 7AL, UK \\ b Department of Analytical Chemistry, Ghent University, Belgium \\ ${ }^{\mathrm{c}}$ Swiss National Museum, Zurich, Switzerland \\ ${ }^{\mathrm{d}}$ Koninklijk Instituut voor het Kunstpatrimonium, Belgium
}

Available online 10 August 2005

\begin{abstract}
This paper describes a new application in cultural heritage and other areas for a highly surface specific analytical technique originally developed for semiconductor research. The technique, ultra-low-energy dynamic secondary ion mass spectrometry (uleSIMS), is microdestructive, but has a sensitivity typically better than 1 atom in $10^{6}$. It can provide an analysis within the top $\mathrm{nm}$, or the top few $\mu \mathrm{m}$ of a surface, and gives chemical fingerprinting as well as atomic composition information. It is complimentary to other near-surface techniques such as SEM-EDX, XRD and electrochemical methods. Here, we describe the use of uleSIMS with SEM and SEM-EDX in a study of the tarnishing of museum silver. We report on the initial stages in the development of reference surfaces for control experiments, and on the data obtained from a lightly tarnished sterling silver test coupon exposed in a museum environment for 2 years. First results from a study of a XVII c. silver fragment, aimed at detecting differences in the tarnish or coating in different areas are also presented. Overall we show that the surface chemistry of all these surfaces is a complex mixture of that due to corrosion, contaminants deposited by solvents, polish media (in an overlayer which may only be a few nm thick), handling and the environment, as well as particulates - both from the environment and from polishes. However, surfaces with different histories show large variations in their uleSIMS spectra and depth profiles, and we attempt to lay the groundwork for the interpretation of these.
\end{abstract}

(c) 2005 Elsevier B.V. All rights reserved.

PACS: $61.72 . \mathrm{Ss} ; 82.80 . \mathrm{Ms} ; 81.65 . \mathrm{K}$

Keywords: Silver tarnish; Cultural heritage; Ultra-low-energy SIMS

\footnotetext{
* Corresponding author. Tel.: +44 2476 523900; fax: +44 2476692016.

E-mail address: m.g.dowsett@warwick.ac.uk (M.G. Dowsett).
} 


\section{Introduction}

Ultra-low-energy dynamic secondary ion mass spectrometry (uleSIMS) is a microdestructive analytical technique developed originally for applications in semiconductor research and development [1]. SIMS in general has the attribute of extremely high analytical sensitivity (detecting impurities at levels down to below 1 part in $10^{6}$ atomic whilst consuming sample volumes in the range $10^{-12}$ $\left.10^{-9} 1\right)$. The technique is divided into two branches distinguished by applications and instrumentation. These are static SIMS [2], which is used to analyze the top mono-atomic or mono-molecular layer, and dynamic SIMS [1] which is used for microvolume sampling and imaging, and depth profiling. Dynamic and static SIMS have been used before in applications related to cultural heritage materials (e.g. [3-6]), and specifically to study silver tarnishing [7] but the application of uleSIMS is new to this area.

In uleSIMS the sample is bombarded by a primary ion beam with an energy $E_{\mathrm{p}} 0.1 \leqslant E_{\mathrm{p}} \leqslant$ $1 \mathrm{keV}$. At such energies, the penetration depth of the primary ions is a few $\mathrm{nm}$, and, in general, the perturbation of the sample chemistry extends only over this range. The main application for the technique has so far been ultra-shallow and ultra-high depth resolution depth profiling of semiconductors. In the work described here, we are pioneering the application of uleSIMS to cultural heritage materials, and also, more generally, for mass spectrometry of the near surface region of inorganic samples - i.e. we are using the technique to generate mass spectra for the purpose of gathering molecular information as well as examining individual masses related to the elemental constituents as in conventional depth profiling.

The potential attributes of uleSIMS used in this way are an ability to combine chemical analysis of surface layers from incomplete monolayers to those which are many microns in thickness, with sectioning of the layer so that it can be sampled at any depth. For example, in the case of a corrosion layer, static SIMS would sample just the top monolayer of the corrosion, whereas uleSIMS can clean away superficial layers which may not be sample-related reaction products, to reveal the corrosion below. However, the interpretation of the resulting spectra is in its infancy, and the extent to which chemical information is preserved by the dynamic SIMS conditions has to be tested on a case by case basis. The technique should be seen as complimentary to electrochemical and other methods [7-10].

The fact that SIMS can provide a fairly comprehensive, very high sensitivity analysis with high spatial resolution whilst consuming a very small sample volume is potentially attractive in the cultural heritage field. However, SIMS shares the limitation common to many surface analytical and electron microscopy techniques, that only a small sample can be accommodated on the analytical stage. Actually, this is an instrument engineering limitation, not a fundamental one in the type of instrument used here: future instrumental development should allow artefacts of a few $\mathrm{cm}$ linear dimensions to be analyzed, reducing the need for sample-taking. Moreover, it is often the case that corrosion and passivation/protection studies are carried out on test coupons of modern alloys or simulants. In this case, these can be cut or preconfigured to fit existing sample mounts.

In this paper we discuss the first examples of the application of uleSIMS to problems related to the tarnishing of museum silver, including surface preparation using various polishing media, the production of reference materials, and a study of dark and light tarnish areas on a XVII c. silver fragment.

\section{Experimental overview}

The samples used in this study are of three types: (i) sets of reference surfaces produced by vacuum evaporation of silver onto silicon, and polishing of pure silver sheet; (ii) one of a set of pure and sterling silver coupons, polished with "Tripoli", a commercial metal polish (see Section 3 ) and then exposed for 2 years in various locations in the Swiss National Museum (these samples also had regions which had been protected with polydimethyl siloxane (PDMS) [14] but work on these is incomplete and will be reported elsewhere); (iii) a fragment of XVII c. silver which was part of 
a restoration of the XII c. Shrine of St. Domitianus, from Notre-Dame Kathedral of Huy, and supplied by Koninklijk Instituut voor het Kunstpatrimonium, Belgium. The samples are described in more detail in Section 3.

The analyses were performed in an Atomika 4500 SIMS instrument [11] equipped with ultralow-energy ion guns delivering $\mathrm{Cs}^{+}$and $\mathrm{O}_{2}^{+}$beams [12]. Positive secondary ion data were obtained with 10 or $20 \mathrm{nA}$ of $500 \mathrm{eV} \mathrm{O}_{2}^{+}$incident at $30^{\circ}$ to the surface normal, whilst negative ions were sputtered using $11 \mathrm{nA}$ of $500 \mathrm{eV} \mathrm{Cs}^{+}$incident at $50^{\circ}$. As a rule, the beams were scanned over a $1 \mathrm{~mm}$ square to reduce the erosion rate and enhance the sampling of the surface. In contrast, spectra corresponding to bulk alloy composition were taken with stationary beams of $50 \mu \mathrm{m}$ diameter at beam currents up to $80 \mathrm{nA}$ (spot mode) at the bottom of a previous SIMS crater. For all of the spectra, the mass range 0-250 Da was scanned at a rate of $10 \mathrm{Da} / \mathrm{min}$.

Additionally, uleSIMS depth profiles were measured under similar primary beam conditions to the spectra. Strictly speaking one should perhaps refer to these as "ion beam cleaning curves" rather than depth profiles, partly because the starting surfaces are not close to atomically flat (the norm when using ultra-low-energy depth profiling), and also since much of their interesting behaviour is in an extended transient region [13]. Their value lies in the comparison they offer between different surfaces.

Data were also taken by SEM and SEM-EDX using a $20 \mathrm{keV}$ beam and compared with the SIMS.

\section{Samples and sample preparation}

The reference surfaces were made in one of two ways:

(i) Silver wire ( $1 \mathrm{~mm}$ diameter, $99.9 \%$ pure) was evaporated in a vacuum of $2 \times 10^{-7} \mathrm{mbar}$ from a molybdenum boat in a commercial evaporator onto semiconductor grade silicon (100) substrates. Layers were approximately $1.8 \mu \mathrm{m}$ thick and were reasonably adherent, although care was needed to prevent tearing of the silver when cleaving the wafer for mounting in the SIMS instrument.

(ii) In preliminary experiments, diamond polishes were found to leave the least intrusive residues compared to alumina and silica. Oil-based polish was avoided because of the complexity added to the surface spectra by residual hydrocarbons. Silver plates cut from 99.95\% pure, $1 \mathrm{~mm}$ thick, silver sheet were mechanically polished in a custom jig using a Minimet polisher (Buehler Ltd.). Water based colloidal diamond (Kemet Ltd.) was employed in three polishing stages $(6 \mu \mathrm{m}$, $1 \mu \mathrm{m}$ and $0.1 \mu \mathrm{m}$ ) of $8 \mathrm{~min}$ each, with ultrasonic cleaning in deionised (DI) water between each stage to remove the previous polish residue. Because DI water is very reactive and will oxidise the silver surface, it was not used after the final polish. Mounting wax was removed with warm propan-2-ol. Finally there were two ultrasonic cleaning stages (5 min) using fresh "Ultra" grade propan2-ol and a clean vessel for each, followed by drying in a class 100 laminar flow cabinet. Dry plates were stored in a membrane box.

The sample from the Swiss National Museum was a sterling silver which had been polished using the commercial polish "Tripoli". This is a complex substance with no standard composition, based historically on crushed siliceous limestone. Modern polishes are typically artificial, proprietary mixtures designed to mimic the limestone properties. Its principal constituents are silica, alumina (or alumino-silicates), and calcium oxides and carbonates. It contains a large range of inorganic compounds including those of titanium and iron. In this report, we concentrate on data from a tarnished surface which had a blue-black appearance. After initial polishing, the $0.5 \mathrm{~mm}$ thick silver plate was placed for 2 years on a cloth surface in a glass cabinet. For SIMS analysis a piece was cut from the plate using carefully cleaned metal shears to prevent the generation of metal swarf and the transfer of oil. Prior to SIMS analysis it was exposed to a nitrogen gas jet to remove any loose dust. 
The XVII c. silver fragment (Fig. 1) was about $22 \mathrm{~mm} \times 12 \mathrm{~mm}$ and contained two nail holes. It was far from flat, being both crumpled and bent on a mm scale. No cutting and minimal distortion was permitted when mounting in the SIMS sample holder. Since the sensitivity and reproducibility of SIMS data are very dependent on sample topography, this was a potential problem. The high depth of focus of the secondary ion optics in the quadrupole SIMS instrument was used to advantage here. The fragment featured a dark band parallel to the long edge, with lighter tarnish elsewhere. The uleSIMS was used to assess any chemical differences between the light and dark regions in order to help determine whether the dark band was a deliberately applied coating or a region of heavier tarnish. This sample was ultrasonically cleaned in "Ultra" grade propan-2-ol for 10 min before loading into the instrument in an attempt to remove loose dust, and any organics from handling.

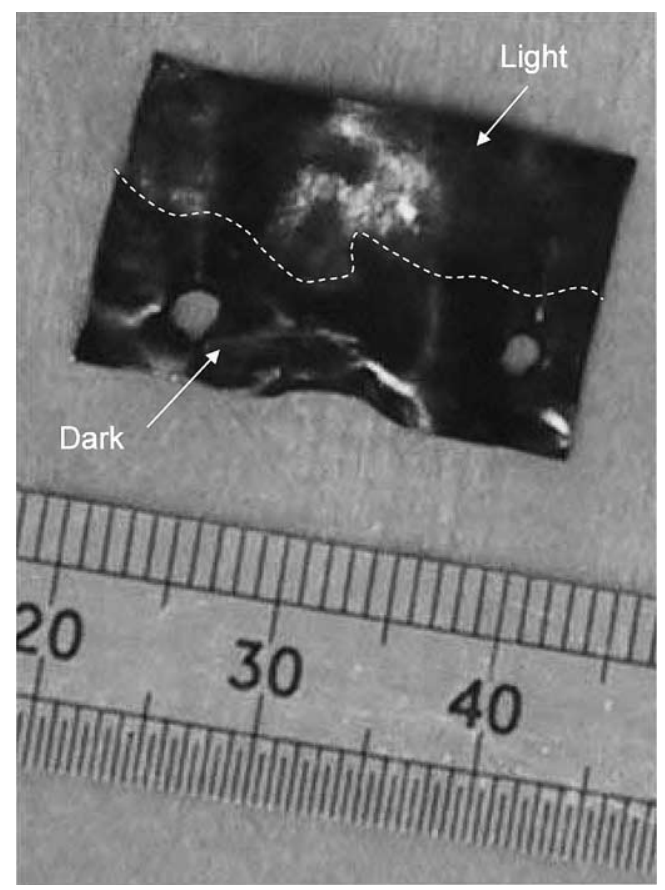

Fig. 1. A fragment of silver from a XVII c. restoration of the XII c. Shrine of St. Domitianus in the Notre-Dame Kathedral of Huy. (Koninklijk Instituut voor het Kunstpatrimonium, Belgium.) The dashed line marks the approximate boundary between the light and dark areas.

\section{Results and discussion}

For comparison, all the spectra presented below have been normalized to a beam current of $10 \mathrm{nA}$. Peaks are identified (where possible) on the basis of isotopic fingerprints (i.e. groups of peaks whose relative intensities are characteristic of combinations of the naturally occurring isotopes of the elements concerned), intercomparison between surfaces (including silicon, differently prepared silver, and silver stock used to make the samples), behaviour of peak groups during cleaning, and other factors. The line spectra were produced from the original mass spectra by averaging across nine data points on the peak.

Peaks identified with bold type in the positive ion spectra (e.g. 16, 32, 123, 125, 230, .. Da) are formed partly by interaction between the surface and the primary $\mathrm{O}_{2}^{+}$ion beam. Additionally the Cs peak at $133 \mathrm{Da}$ comes from an extremely small level of surface diffused $\mathrm{Cs}$ from the negative ion analysis.

Overall, it needs to be remembered that SIMS spectra are indicative of the chemical composition of the top $\mathrm{nm}$ of the sample at the moment of acquisition, whereas SEM EDX gives the average composition of a near surface volume extending around $4 \mu \mathrm{m}$ inwards. The SIMS data in particular contain signals from three different sources: (i) bulk constituents with levels well below the detection limit of EDX, but often giving a strong SIMS signal, (ii) quasi-uniform superficial layers of corrosion and contamination, (iii) particulate contamination from the environment and from polish residues.

\subsection{Reference surfaces}

The reference surfaces represent a first attempt to identify the problems involved in producing and analyzing materials with a known starting condition for either subsequent reaction in a corroding environment, or direct comparison with more complex "real" surfaces. Since SIMS exhibits a high differential sensitivity across the periodic table (relative peak heights for different elements cannot be directly related to relative concentration) and highly surface specific chemical frag- 
mentation patterns, the development of reference materials is exceptionally important. Except for heavily sputter cleaned samples, the polished and evaporated surfaces showed the lowest contamination levels in the experiments reported here.

The evaporated surfaces are the most idealized as they were produced from high purity wires, and are extremely flat (although they show distinct grain structure on a scale of $\sim 1 \mu \mathrm{m}$ in SEM). The polished surfaces are a step closer to reality since they will exhibit the grain structure of the rolled pure silver or alloy sheet, and will be scratched at least on the micrometer scale. There may also be superficial impurities and inclusions pressed into the surface from the sheet metal production process.
It is worth mentioning at the outset that all surfaces cleaned with abrasive polishes (alumina, silica, Tripoli, diamond) contained residues which were easily detectable using SIMS, even after extensive ultrasonic cleaning. Diamond residues were the least intrusive and contributed the least to the spectra. We intend to detail the work on polish residues elsewhere.

Fig. 2(a) and (b) show, respectively, positive and negative ion spectra from the surface of the evaporated silver (grey lines) and from the polished silver (black lines).

\subsubsection{Positive ion spectra and depth profiles}

Sodium and potassium are extremely high yield elements in positive ion mode, and are common in the environment, so these elements often feature
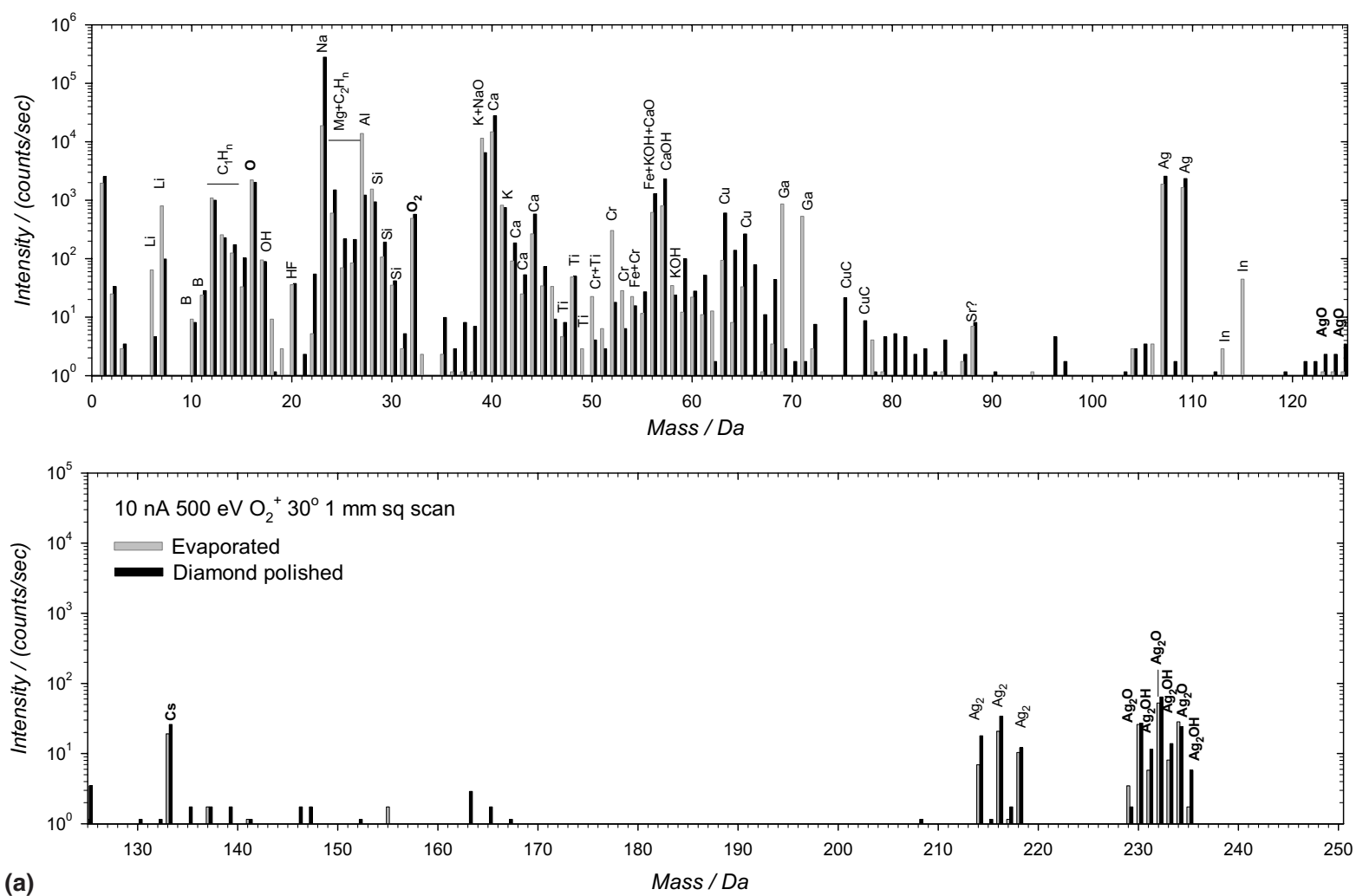

Fig. 2. (a) Positive ion spectra from the surface of a $1.8 \mu \mathrm{m}$ thick silver layer evaporated onto (100) silicon (grey bars) and from a diamond polished surface on pure silver (black bars). Primary beam: $10 \mathrm{nA} 500 \mathrm{eV} \mathrm{O}+$ incident at $30^{\circ}$ to normal and scanned over a $1 \mathrm{~mm}$ square area. (b) Negative ion spectra from the same surfaces. Primary beam: $10 \mathrm{nA} 500 \mathrm{eV} \mathrm{Cs}^{+}$incident at $50^{\circ}$ to normal and scanned over a $1 \mathrm{~mm}$ square area. 

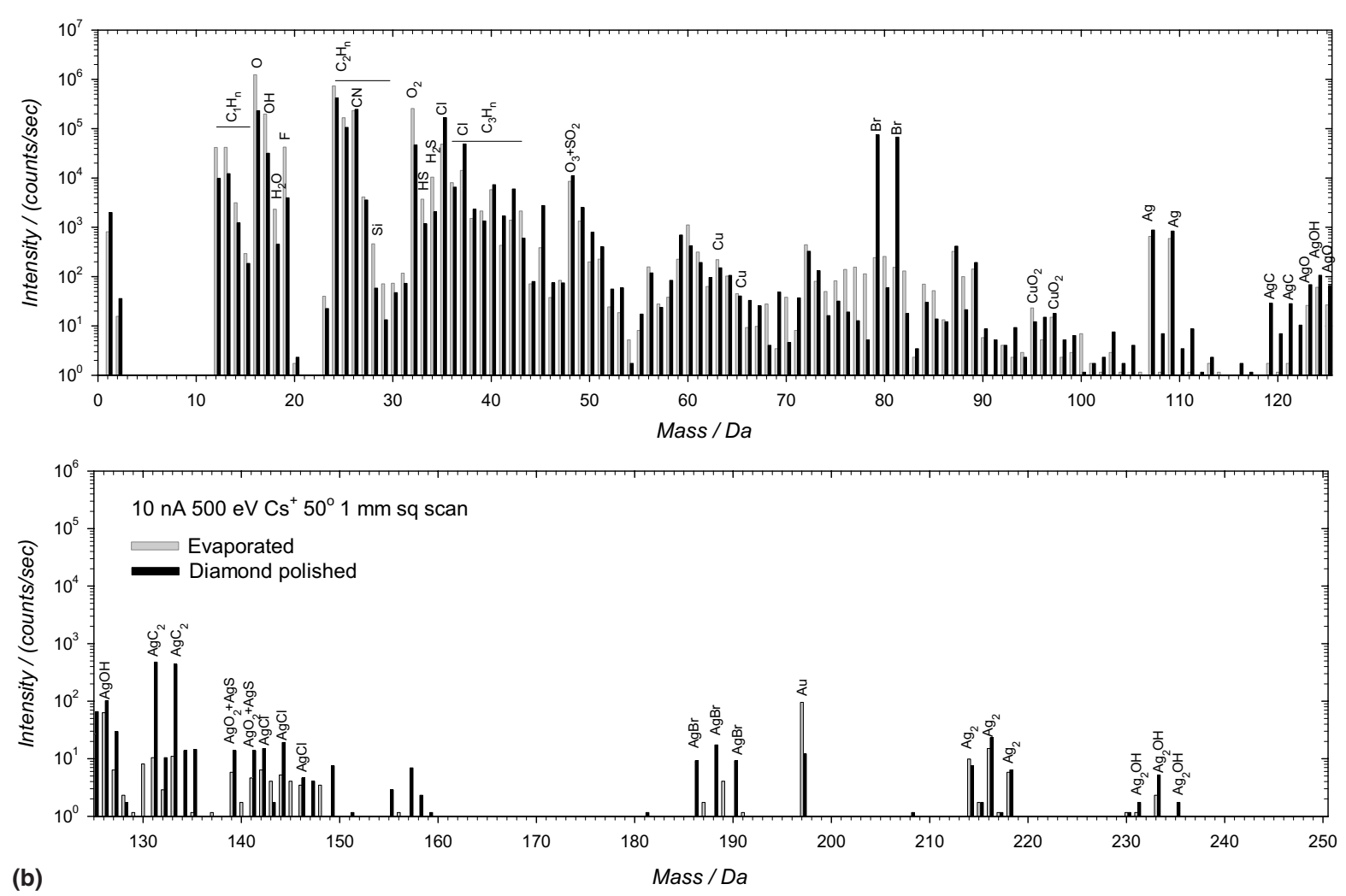

Fig. 2 (continued)

prominently in SIMS spectra from surfaces. However, the Na peak from the polished sample is more than a factor of 10 higher than that from the evaporated sample. Indeed, despite the otherwise generally low levels of contamination, this surface had the highest $\mathrm{Na}$ level of any of those described here. uleSIMS depth profiling (see Fig. 3) showed that the Na level from the evaporated sample decreased slowly into the bulk, whilst that from the polished sample decreased by over an order of magnitude in the first few $\mathrm{nm}$. The high $\mathrm{Na}$ level on the latter is therefore likely to be a residue from the polishing process - probably left as a fairly uniform Na-containing layer in the form of a drying stain.

More surprising is the appearance of $\mathrm{B}, \mathrm{Mg}, \mathrm{Si}$, $\mathrm{Ca}, \mathrm{Ti}, \mathrm{Fe}$ and possibly $\mathrm{Sr}$ at similar levels in both spectra. (Note, however, that peaks identified as $\mathrm{Fe}^{+}$may be $\mathrm{CaO}^{+}-$see discussion on particulate contamination on exposed silicon surfaces below.)
The surface of a cross-section cut with a scalpel through the wire used in the evaporation was analyzed for comparison with the evaporated layer. The spectrum showed the cut surface to be more highly contaminated than the evaporated layer, with the metallic species listed above at levels of 2-5 times higher, and unidentified hydrocarbon fragments at relatively higher levels still. Si and Al levels were around 10 times higher. SEMEDX of the cut and evaporated surfaces showed no signals other than those of silver, putting the average impurity level for elements with atomic numbers above 10 at less than $0.1 \%$. The contamination levels are superficial, therefore, and not representative of the bulk. We will return to a more detailed discussion of their origins later.

A major difference between the spectra of diamond polished and evaporated silver is the occurrence of relatively higher levels of $\mathrm{Li}, \mathrm{Al}, \mathrm{Cr}, \mathrm{Ga}$ and In on the evaporated surface. Ga and In are 

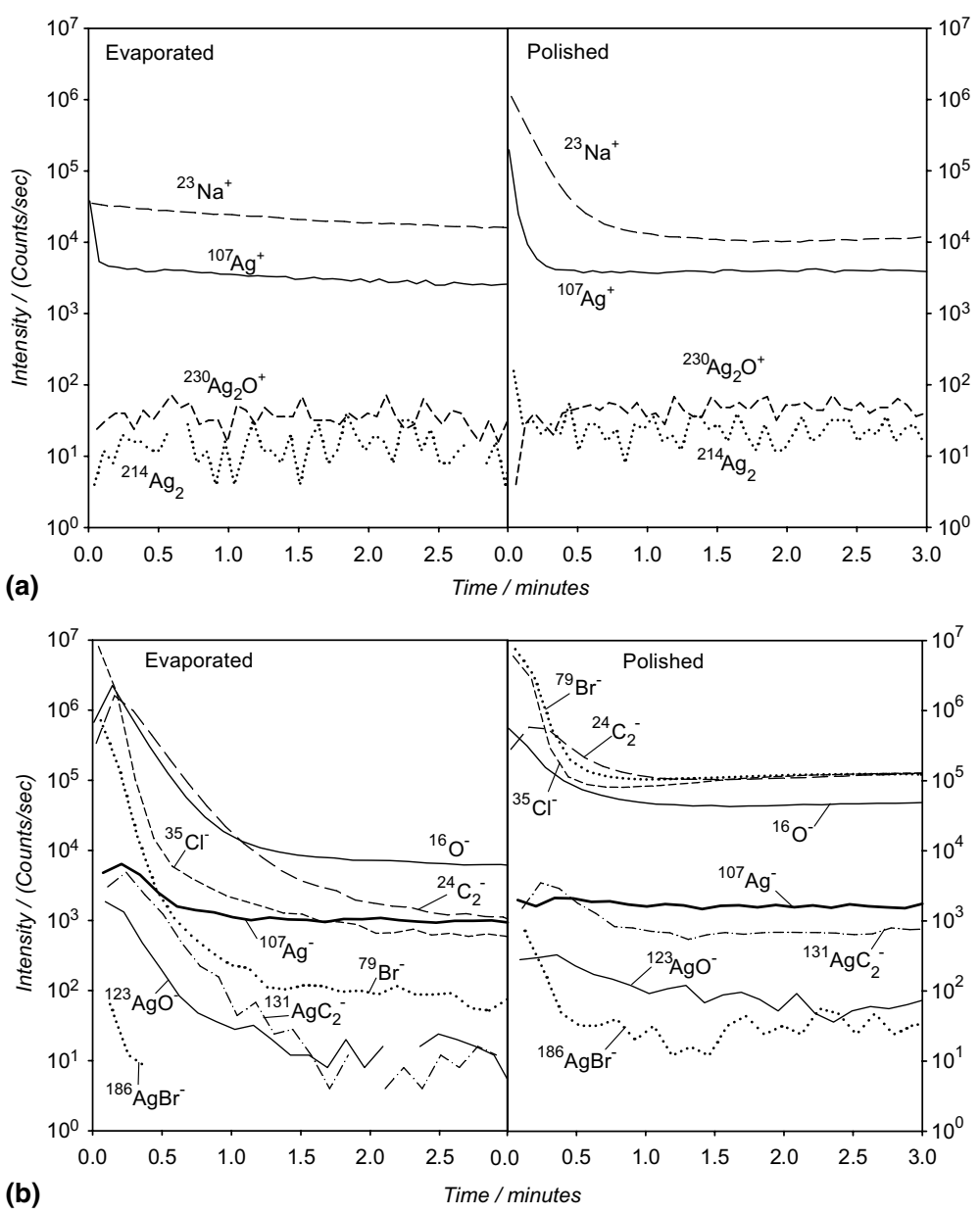

Fig. 3. (a) Ultra-low-energy positive ion depth profiles (or cleaning curves) from the top few nm of the evaporated (left panel) and diamond polished (right panel) surfaces. Note the large decrease for $\mathrm{Na}$ and $\mathrm{Ag}$ signals in the first 0.5 min for the polished layer. Primary beam: $10 \mathrm{nA} 500 \mathrm{eV} \mathrm{O}_{2}^{+}$incident at $30^{\circ}$ to normal, $1 \mathrm{~mm}$ square area. (b) Negative ion profiles from the same surfaces. In contrast to the positive ion profiles, many surface species in the polished sample remain around a factor of 10 higher at the end of the profile. Primary beam: $10 \mathrm{nA}, 500 \mathrm{eV} \mathrm{Cs}^{+}, 50^{\circ}$ to normal, $1 \mathrm{~mm}$ square area.

not observed on the wire section and are low level contaminants arising from previous use of the evaporator. These are elements with very high positive ion yields under oxygen bombardment, and the signals observed probably correspond to less than $0.01 \%$. $\mathrm{Li}$ and $\mathrm{Cr}$ are at the same levels observed on the cut wire surface, whilst $\mathrm{Al}$ is a factor of $\sim 10$ lower. Again, these are all high yield elements, and the signals correspond to $<0.01 \%$ concentration. The systematic behaviour of contaminants in and on evaporated layers needs to be established - especially considering the possi- bility of impurity fractionation during evaporation and resulting concentration gradients in the layers.

Finally we should mention the $\mathrm{Cu}$ level. Copper is a common impurity in silver and is present in the stock at about $500 \mathrm{ppm}$ (supplier's data). However, the levels of $\mathrm{Cu}$ cannot be quantified from these surface spectra because the yields are strongly modified by the surface chemistry.

Fig. 3(a) shows positive uleSIMS profiles giving a comparison between the polished and evaporated signal behaviour for $\mathrm{Na}, \mathrm{Ag}, \mathrm{Ag}_{2}$ and $\mathrm{Ag}_{2} \mathrm{O}$ as the top few $\mathrm{nm}$ of the sample are 
sputtered away by the analyzing beam. Corresponding signals in each profile tend to the same values after $3 \mathrm{~min}$, but the very high initial values of $\mathrm{Na}^{+}$and $\mathrm{Ag}^{+}$, and their sharp decrease over the first $0.5 \mathrm{~min}$. on the polished surface is indicative of a thin, relatively uniform contaminant layer left as a polish residue.

\subsubsection{Negative ion spectra and depth profiles}

Fig. 2(b) shows the negative ion spectra from the evaporated and diamond polished surfaces. Much of the spectrum shows common levels of contamination in the form of unidentified hydrocarbon fragments, although the evaporated layer shows higher levels between 70 and $80 \mathrm{Da}$ which may be due to the vacuum in the evaporator. There are, however, some obvious differences between the two surfaces. The evaporated surface is richer in $\mathrm{O}, \mathrm{OH}$, possibly $\mathrm{S}$, and $\mathrm{Au}$. The last is a bulk impurity, perhaps enriched in the near surface region during the evaporation of the wire or arising from previous use of the evaporator. It is not detectable in EDX, and so is at a level below $0.1 \%$.

The diamond polished layer shows 3.5 times the intensity for $\mathrm{Cl}$ and $>300$ times that for $\mathrm{Br}$ compared to the evaporated layer, and levels of $\mathrm{AgCl}$ and $\mathrm{AgBr}$ which are 5-8 times higher. The halogens may come from the propan-2-ol, the polish medium, or might be impurities leached from some unidentified source (containers, the part-polished silver surface) and deposited by the deionised water. To determine which of these is the source, polishing protocols where the ultrasonic cleaning was done entirely in propan-2-ol were carried out. These did not show the anomalously high $\mathrm{Br}$ and $\mathrm{Cl}$ levels, so we believe that the water was the cause of the contamination, or was ineffective in removing polish residue. It is tempting to associate the halogen and sodium levels and assume that there is a nm-scale $\mathrm{NaCl} / \mathrm{NaBr}$ drying stain. However, the sub-surface behaviour of $\mathrm{Br}$ and $\mathrm{Cl}$ observed in negative ion profiles (see below) is not consistent with this, and the $\mathrm{Br}$, at least, is bonded with the silver.

Another notable difference between the polished and evaporated surfaces is the 100 times higher levels of $\mathrm{AgC}$ and $\mathrm{AgC}_{2}$ seen on the former.
This is likely to be due to embedded polish particles which may have undergone surface reaction with the metal, or simply be coated with a thin metal film. Silver polished with alumina showed similar peaks identified as AgAl in the spectra.

Fig. 3(b) shows negative ion profiles for various species from the spectra. For the evaporated surface, the positive and negative data together are consistent with a surface layer a few nm thick containing silver oxides and halides. All species associated with oxidation or contamination decrease by factors of between 100 and 10,000 in the first minute (corresponding to the removal of $1-3 \mathrm{~nm}$ under these conditions). In contrast, although contaminant species show some initial decrease on the polished surface, their steady-state intensities remain 10-100 times higher at the end of the profile in comparison with the evaporated sample. Only $\mathrm{Ag}^{-}$tends to a similar level. The persistence of species such as $\mathrm{C}_{2}^{-}$and $\mathrm{AgC}_{2}^{-}$is indicative of diamond particles embedded in the surface. It is clear that some other residue from the polishing operation is also present. This may be in the form of small crystals rather than a continuous layer (explaining the persistence of species such as $\mathrm{Br}^{-}, \mathrm{AgBr}^{-}$and $\mathrm{Cl}^{-}$during sputtering), and SEM work is under way to see if this is the case.

\subsection{Tarnished sterling silver}

This surface varied between dark straw and blue-black in appearance. However, the silver colour of the surface was restored by the sputter cleaning effect of the SIMS analysis, despite the low ion dose. This shows that the tarnish layer averages only a few nm thick. Nevertheless, contaminant signals persisted in spectra taken after the surface was visually clean.

Fig. 4(a) and (b) show positive and negative ion spectra from the tarnished layer and from the same surface after ion cleaning with the analyzing beam. This sample was part of a larger study, and is presented here because it is an example of a surface exposed to atmospheric contaminants in a museum in a modern city (Zurich). Sterling silver is an alloy containing approximately $8 \%$ copper so that $\mathrm{Cu}, \mathrm{Ag}$, and peaks containing combinations of the two appear in the spectra. 

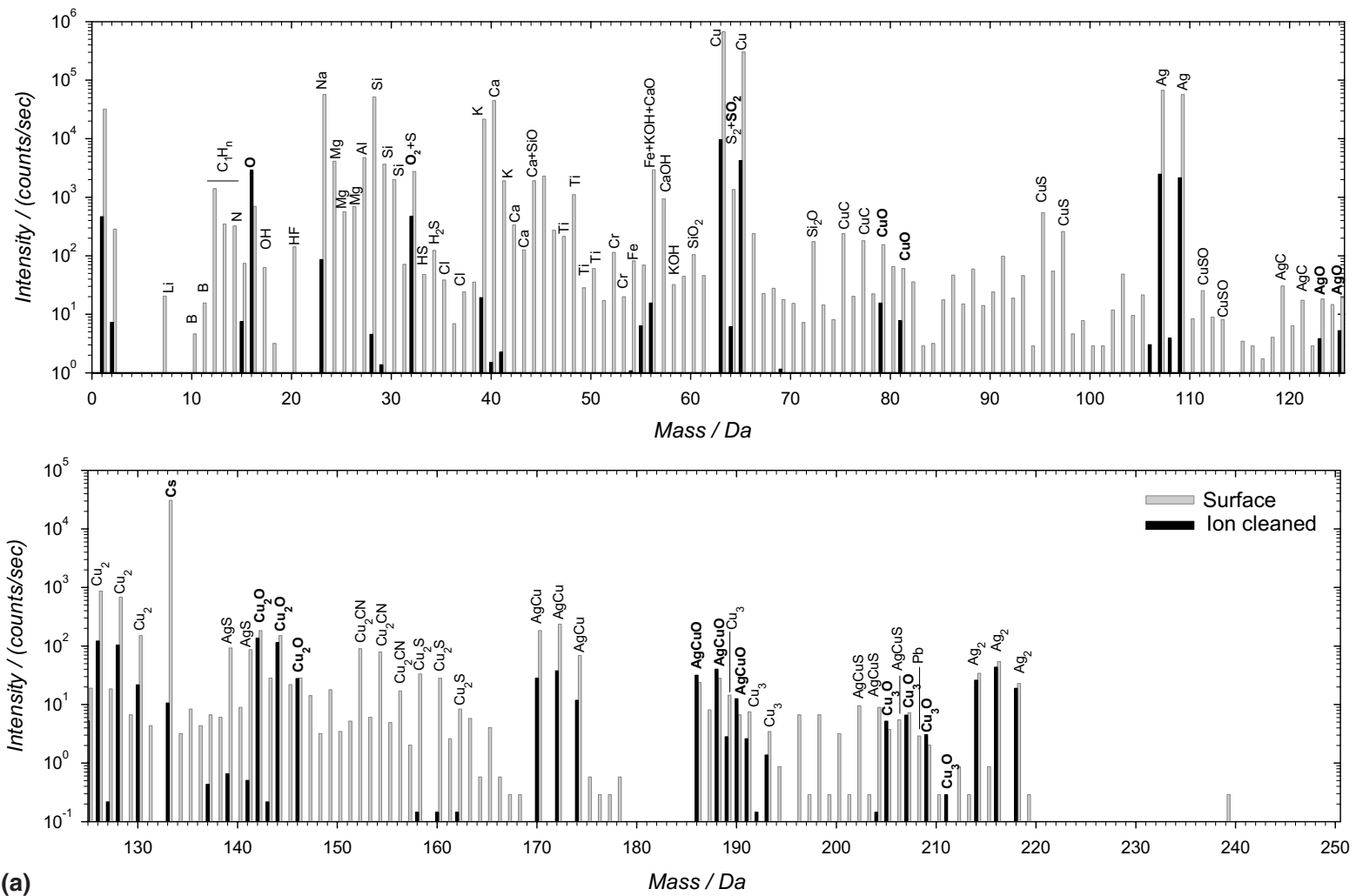

Fig. 4. Spectra from a sterling silver test coupon polished with Tripoli and exposed in a glass cabinet for 2 years in the Musée Suisse. (a) Positive ions from the tarnish surface (grey bars) and from a surface sputtered $35 \mu \mathrm{m}$ deep into the sample (black bars). Primary beam: $10 \mathrm{nA} 500 \mathrm{eV} \mathrm{O}_{2}^{+}$incident at $30^{\circ}$ to normal, $1 \mathrm{~mm}$ square area on tarnish, spot mode on the sputtered surface. (b) Negative ion spectra from the tarnish surface (grey bars) and following a cleaning dose of $1.8 \times 10^{18}$ ions cm ${ }^{-2}$. Primary beam: $10 \mathrm{nA} 500 \mathrm{eV} \mathrm{Cs}{ }^{+}$ incident at $50^{\circ}$ to normal, $1 \mathrm{~mm}$ square area on tarnish, spot mode in bulk.

The positive spectrum from the tarnish (grey bars) shows very high levels of $\mathrm{Mg}, \mathrm{Si}, \mathrm{Ti}, \mathrm{Cr}$ and Fe compared to the polished or evaporated samples. The $\mathrm{Ca}$ and $\mathrm{K}$ levels are similar, $\mathrm{Na}$ and $\mathrm{Li}$ are significantly lower. There are also fingerprints of the corrosion products in the tarnish which we will describe below. Other peaks to note are $\mathrm{SiO}, \mathrm{SiO}_{2}, \mathrm{Si}_{2} \mathrm{O}$, which, despite the use of $\mathrm{O}_{2}^{+}$, and by comparison with peak ratios from pure silicon, we believe to be indicative of the presence of silica on the surface.

The spectrum from the ion cleaned surface (black bars) was obtained after sputtering $35 \mu \mathrm{m}$ into the sample. This was necessary to get through the particulate contamination and the rolled "skin" on the silver and into a region representative of the bulk composition. The result is the cleanest silver spectrum we have observed, and contains only beam-related species $\left(\mathrm{O}, \mathrm{O}_{2}\right.$ and $\mathrm{Cu}_{m} \mathrm{O}_{n}, \mathrm{Ag}_{m} \mathrm{O}_{n}, \mathrm{AgCuO}$ ), small $\mathrm{Na}$ and $\mathrm{K}$ peaks, and the $\mathrm{Cu}$ and $\mathrm{Ag}$ peaks themselves. The following features are typical of a SIMS analysis: (i) the signal strengths for $\mathrm{Ag}$ and $\mathrm{Cu}$ from the tarnished surface are around 50 times higher because of the ion yield enhancement due to their oxidised chemical state, (ii) the $\mathrm{Cu} / \mathrm{Ag}$ signal ratios on the tarnished and cleaned surfaces are 10 and 3.9, respectively. This is because $\mathrm{Ag}$ is more noble than $\mathrm{Cu}$ and the promotion of its ion yield by general oxidation in the tarnish or specifically from the analyzing beam is far less. Note that the relative ionization probabilities can only be calculated approximately from these data because of the natural variation of $\mathrm{Cu}$ 

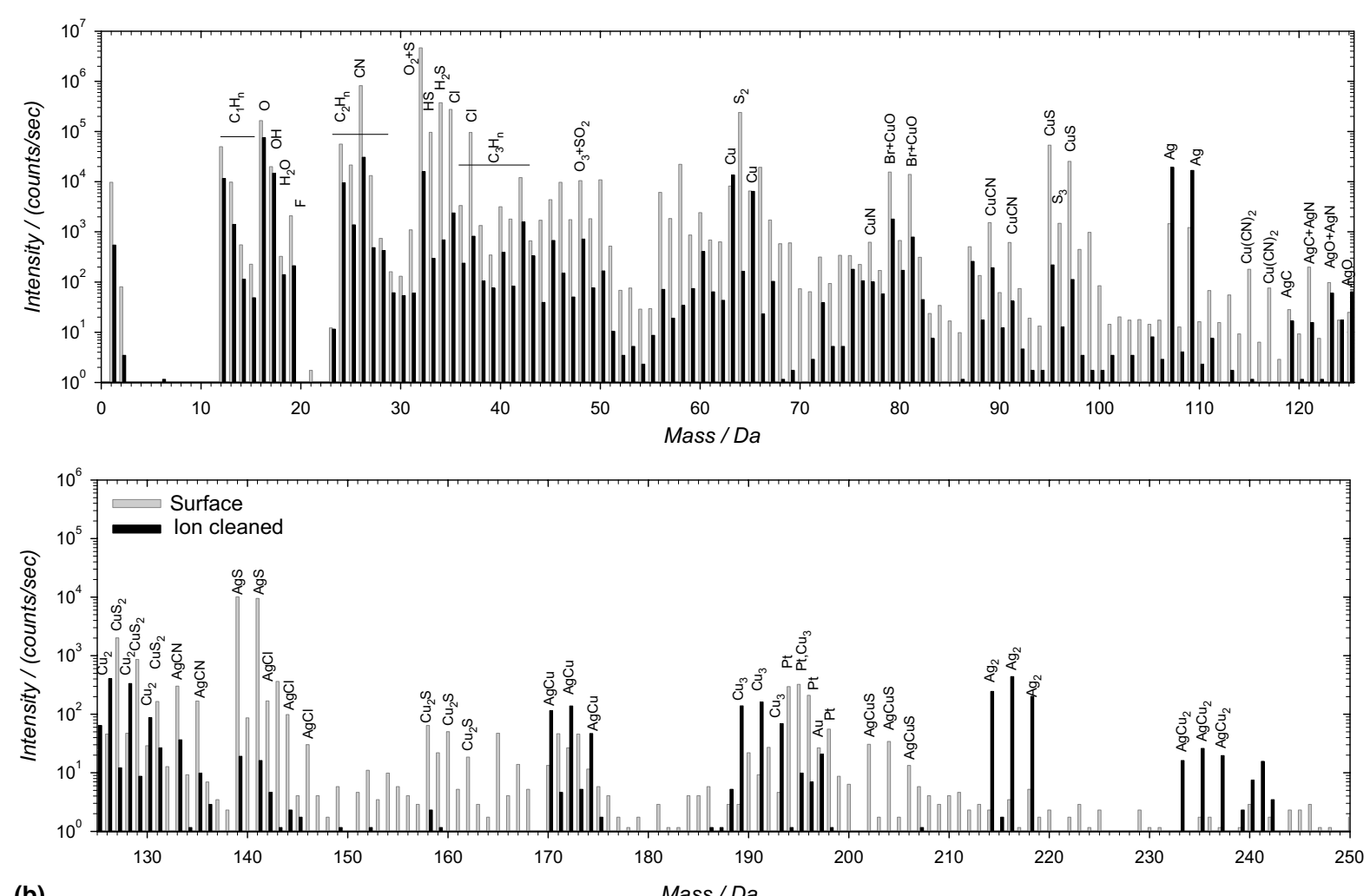

(b)

Fig. 4 (continued)

content across grains, and because EDX showed an inhomogeneous enrichment of $\mathrm{Cu}$ of up to $48 \%$ on the bottom of deep oxygen-sputtered craters. Nevertheless we find the ionization probability for $\mathrm{Cu}$ relative to $\mathrm{Ag}$ to be $\sim 44$ from the clean surface.

We believe that the very high levels of metallic and silicon contamination observed in the tarnish spectrum are indicative of polish residue from the Tripoli. To investigate this further, the surfaces were studied in SEM and found to be rich in particulate contamination. The $1-3 \mu \mathrm{m}$ particles tended to be in clusters numbering 10-20. Fig. 5 (inset) shows such a cluster on the bottom of a lightly sputtered region (in a pure silver sample from the same set) with SEM-EDX from the cluster showing it to be the origin of the $\mathrm{Mg}, \mathrm{Al}, \mathrm{Si}, \mathrm{Ca}$ and Ti signals at least, and possibly contributing to the $\mathrm{O}$ and $\mathrm{C}$ containing species (in both positive and negative spectra). In order to test the tendency for polish residue to contribute to the data in this way we hand-polished a pure silver sample with $1 \mu \mathrm{m}$ alumina powder suspended in de-ionized water for $10 \mathrm{~min}$ using a lint-free pad. Alumina is known to be hard to remove from the silver surface [9]. The sample was then ultrasonically cleaned in propan-2-ol. Similar particle clusters to those described above, at a density of $\sim 12$ clusters per $10 \mu \mathrm{m}$ square were observed. Positive and negative ion spectra from this surface contained strong signals for $\mathrm{Al}\left(\sim 10^{7} \mathrm{cps}\right)$ and $\mathrm{Al}_{m} \mathrm{O}_{n}$ complexes. Further tests using different polishes are in progress.

Fig. 4(b) shows the negative ion data from the tarnished surface (grey bars) and from the underlying surface cleaned with a $\mathrm{Cs}^{+}$dose of $1.8 \times$ $10^{18}$ ions $\mathrm{cm}^{-2}$ (black bars). (It was not possible to measure the crater depth in this case.)

The intense peak at $32 \mathrm{Da}$, accompanied by strong peaks at 26, 33, 34, 35 and $37 \mathrm{Da}$ are 


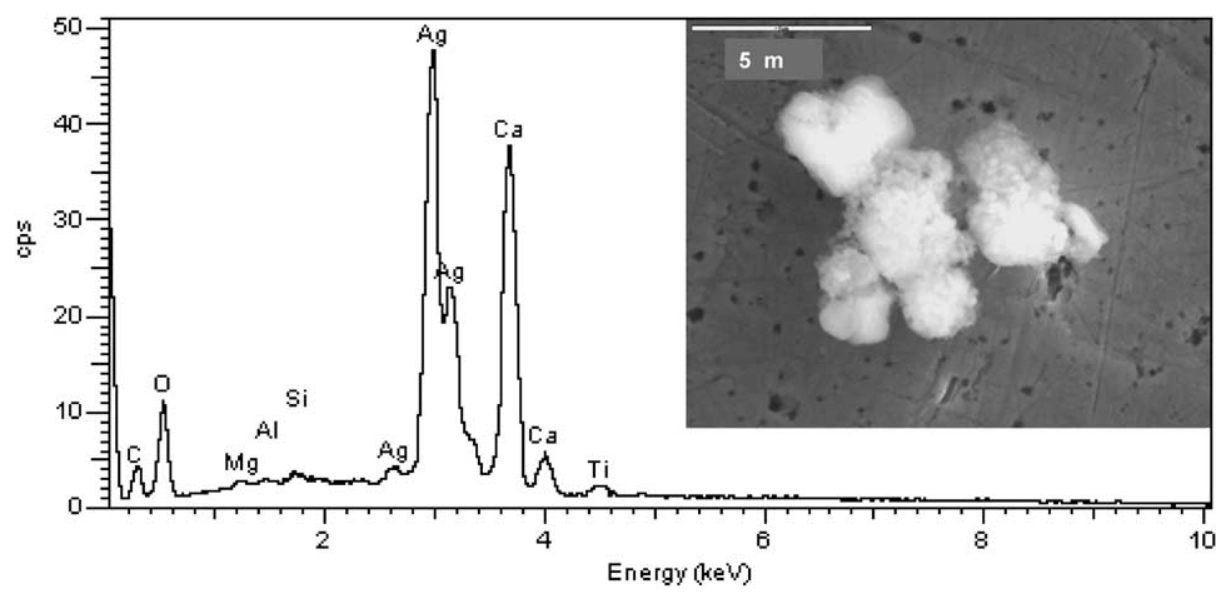

Fig. 5. Lightly sputtered region on the sterling silver sample: SEM-EDX spectrum of the particle cluster shown in the inset. The composition strongly suggests that this is a residue of the Tripoli polish. (SEM $20 \mathrm{keV}$, normal incidence.)

characteristic of the tarnished surface. These are a factor of 5-50 greater than those observed on the polished or evaporated samples. In supporting studies we noted that fresh silver surfaces exposed to air yielded negative ion spectra with the ratio of the peak at $32 \mathrm{Da}$ to that at $16 \mathrm{Da}\left({ }^{16} \mathrm{O}^{-}\right)$being $\sim 0.1$. This surface, and others like it, have 32/16 ratios which are typically $\sim 10$. We associate the high $32 / 16$ ratio with the existence of sulphur in the surface, consistent with the expected formation of silver and copper sulphides in the tarnish. The low 32/16 ratio we associate with oxide formation. Many other peaks in both the positive and negative ion spectra are fragments of silver, copper and mixed sulphides. We also observe cyanides and chlorides whose precursor peaks are at 26 and 35, $37 \mathrm{Da}$. Finally, the spectrum contains a forest of hydrocarbon fragments.

On the ion cleaned spectrum we see a decrease of $\sim 500$ times in the sulphur containing peaks, with an increase in $\mathrm{Ag}^{-}$and $\mathrm{Cu}, \mathrm{Ag}$ and $\mathrm{Ag}-\mathrm{Cu}$ clusters of at least a factor of 10. In general, an increasing level of metal clusters in negative ion mode seems to indicate increasing levels of pure metal in the analyzed area [4]. Although there are large decreases in the range 10-100 times in most of the contaminant associated peaks, the hydrocarbons seem to be resistant to removal, suggesting that they partly originate from surface particles.
The tarnished surface yielded a Pt isotopic signature starting at $194 \mathrm{Da}$ with $\mathrm{Au}$ at $197 \mathrm{Da}$. The former is superficial and cleans away, and almost certainly came from the surface condensation of a Pt-containing organic catalyst carried in a volatile solvent. This was used in the deposition and curing of the PDMS film on the other half of the coupon to that from which this sample was cut. However there was no obvious sign of PDMS itself on this surface.

\subsection{Comments on the nature of the surface contamination}

SIMS shows a rather complex picture of surfaces covered by a mixture of tarnish (predominantly sulphide, but with chlorides and cyanides) and organic and inorganic impurities. The relative levels of these are hard to establish using SIMS, and much of what is seen is undetectable by EDX, because either the atomic number is too low, or the average level in the sampling volume is too low. Further experiments with X-ray photoelectron spectroscopy (XPS), which has a lower sensitivity than SIMS (but which shares a similar surface specificity) and with surface X-ray diffraction are planned. Nevertheless, it is clear that three forms of contamination are present: superficial surface films which are not the result of reactions with the sample, corrosion products, 
and particulates. Furthermore the contaminants have a number of sources: chemical reactions with oxidising species in the environment and adsorption of environmental hydrocarbons - these are usually what one wants to study, particulates and other contaminants arriving from the environment during the exposure or the preparation process, and contamination acquired between the end of the experiment and the analysis. A particular concern here is the fact that all surfaces are exposed to organic dust often of human origin, and inorganic dusts - in the form of airborne ash, plaster, concrete and so on (and these would look very like polish residue, unless the polish was a pure, single substance). Of course, this problem is not unique to the samples analyzed here, but SIMS is probably more sensitive to its presence than any other analytical technique.
As a test, we analyzed the surfaces of very high purity silicon wafers (bulk impurity levels below SIMS detection limits) after ultrasonic cleaning in $99.5 \%$ and $99.7 \%$ pure propan-2-ol (levels typical of high purity laboratory solvents). Alkali metal and calcium contamination was easily seen on these surfaces with the $\mathrm{Li}$ and $\mathrm{K}$ intensities 25 times higher for the purer solvent and $\mathrm{Na} 5$ times higher for the other. Peaks indicative of calcium were also observed, and these were reduced by successive ultrasonic cleans in fresh solvent. These data suggest that, for this case, the solvents were the source of the alkali metals (despite their purity), but dust was the source of the calcium signal. It should be remembered that an evaporating, high purity solvent layer perhaps $\sim 100 \mu \mathrm{m}$ thick can leave behind a film of non-volatile contaminant a few nm thick.
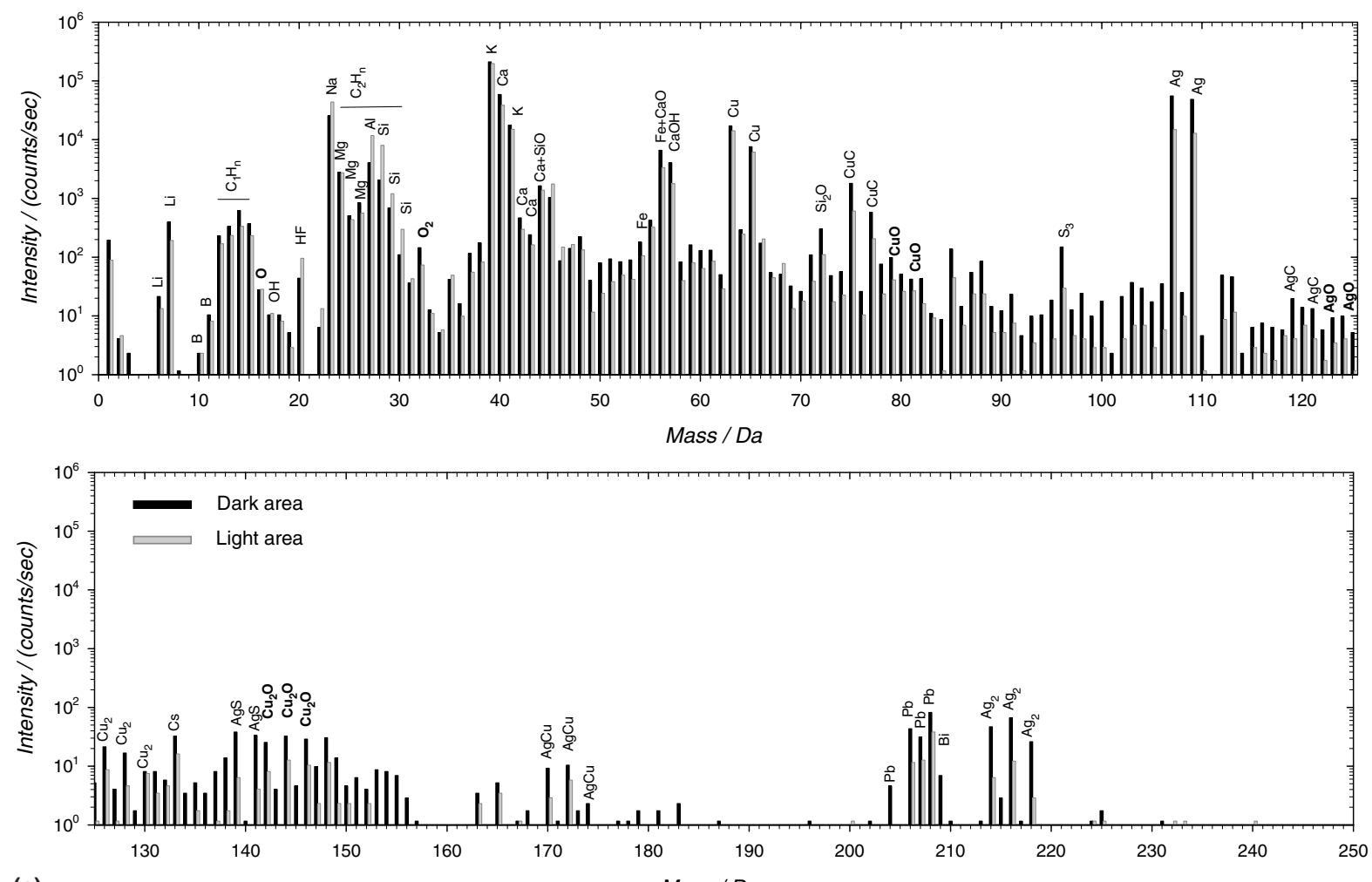

(a)

Mass / Da

Fig. 6. (a) Positive ion spectra from dark and light regions on the XVII c. silver fragment, shown by black and grey bars respectively. Primary beam: $10 \mathrm{nA} 500 \mathrm{eV} \mathrm{O}_{2}^{+}$incident at $30^{\circ}$ to normal, $1 \mathrm{~mm}$ square area. (b) Negative ion spectra. In this case the crumpled nature of the surface meant that comparable data from the light and dark regions had to be taken from a $300 \mu \mathrm{m}$ square region. Primary beam: $10 \mathrm{nA} 500 \mathrm{eV} \mathrm{Cs}^{+}$incident at $50^{\circ}$ to normal. 

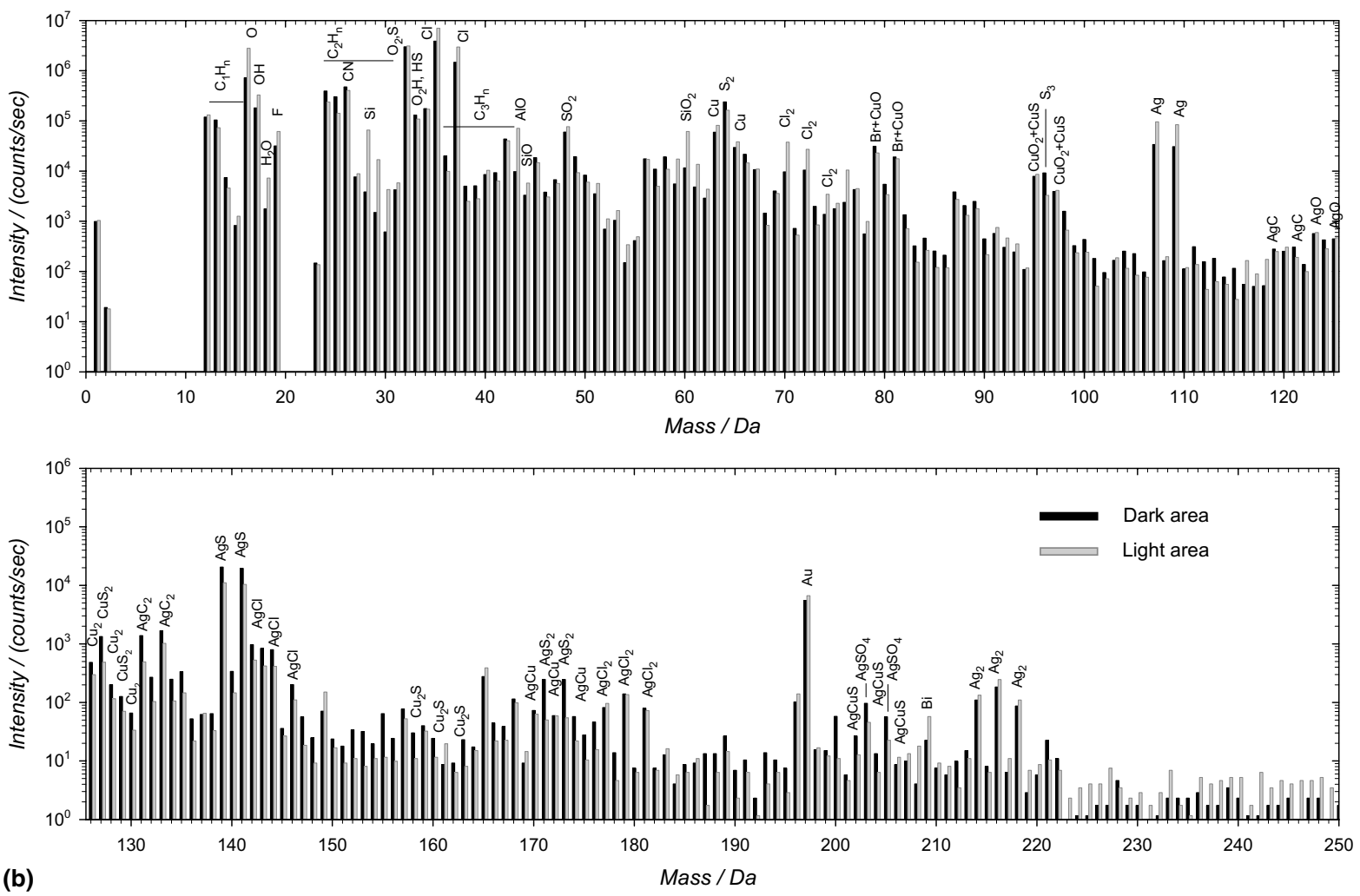

Fig. 6 (continued)

\subsection{XVII c. Silver fragment}

SIMS spectra from this sample are shown in Fig. 6(a) and (b) where the black and grey bars show, respectively, the spectra from the dark and light regions. In this old silver alloy, $\mathrm{Pb}$ and $\mathrm{Bi}$ are clearly seen in the positive ion spectrum (204-209 Da) and $\mathrm{Au}$ is present as a negative ion at around 100 times the level present in modern sterling or pure silver. There is also a significant amount of $\mathrm{Cu}-$ in the range $0.5 \%$ judging by the signal levels relative to sterling silver. The Si level from the light area is higher, and peaks such as $\mathrm{CuC}$ and $\mathrm{AgC}$ (possible carbonate fragments) and $\mathrm{AgS}$ are higher in the dark region.

The negative ion data (Fig. 4(b)) comes from a $300 \mu \mathrm{m}$ square scan, rather than $1 \mathrm{~mm}$ square because the buckling of the surface in the dark region around the nail holes altered the sensitivity too much for the large area scans to be compared.
Both light and dark areas show a similar level at 32 Da to the Musée Suisse sample, but otherwise the tarnish/contamination fingerprint is quite different. $\mathrm{O}, \mathrm{Cl}$ and (again) Si related peaks are higher from the light area $\left(\mathrm{a} \mathrm{Cl}_{2}\right.$ fingerprint is also seen), with $\mathrm{AgC}_{2}$ and $\mathrm{AgS}_{m}$ stronger from the dark region. In contrast to the other surfaces analyzed, significant $\mathrm{AgSO}_{4}^{-}$signals were observed, and were more intense from the dark region. Again, different from other surfaces, very strong $\mathrm{AgCl}$ and $\mathrm{AgCl}_{2}$ signals were present. These cleaned off very rapidly in the ion beam even for $1 \mathrm{~mm}$ square scans.

The negative ion spectrum is also the richest in hydrocarbon fragments of any described here, up to the highest mass observed, at levels of at least 2-10 times that occurring on the Musée Suisse sample. A final difference between this surface and the latter is that the ion beams left no visible marks on the analyzed area, showing the tarnish and contamination to be significantly thicker. 
The overall picture is of a surface containing mixed sulphides, oxides, and chlorides, with sulphates and carbonates also present. The high levels of hydrocarbons are likely to be due to a very long time of exposure to the environment (unknown), frequent handling, and (perhaps) a succession of direct or indirect applications of organically based polishes. One interpretation of the difference between the light and dark areas is that the higher Si levels on the former are from a silica containing polish, and the dark area simply contains a thicker, more complex tarnish layer. There is no evidence to show that the dark region is a specially applied coating.

\section{Conclusions}

Spectra obtained by uleSIMS from silver surfaces with different histories show large variations due to their differing surface chemistry superimposed on a background of similar fingerprints probably due to preparation and environmental films and dust. Because of the sensitivity of the technique, its surface specificity, and its ability to give molecular fingerprint information, the spectra are typically more complex than those from other methods, and, perhaps, give a better indication of the rich chemistry of these surfaces.

It is extremely difficult to produce reference surfaces which appear clean to SIMS, (this has been known for many years) and the development of methods for producing and maintaining such surfaces is an urgent task which is required to put this type of analysis on a more systematic and quantitative basis. Both vacuum evaporation and diamond polishing show promise in this respect. Exposure of such surfaces to polishes, and to different environments is the key to distinguishing between particulate contamination acquired at different stages in the sample history.

Solvent and deionised water cleaning is apparently a source of surface contamination by way of drying stains (and may, perhaps, promote certain surface reactions). The contaminants are either present in the solvents or dissolved from material on the surface itself and re-distributed when the fluid evaporates.

Further study of the polish residues themselves, to establish their analytical intrusiveness, and their spectral fingerprints is required. Another goal is to produce mechanically stable reference surfaces of specific tarnish products so that their fingerprint spectra can be established. We are experimenting with electrochemical methods of doing this.

Finally, it will be important in future work to both extend the mass range and use high mass resolution (for example in a ToF spectrometer) in order better to identify principal fragments from the tarnish.

\section{Acknowledgments}

The authors are grateful to COST Action G8, UK EPSRC, and Cameca GmbH for their support of this work.

\section{References}

[1] M.G. Dowsett, Appl. Surf. Sci. 203-204 (2003) 5.

[2] A. Benninghoven, Surf. Sci. 299-300 (1994) 246.

[3] J.J. Boon, K. Keune, J. van der Weerd, M. Geldof, J.R.J. van Asperen de Boer, Chimia 55 (2001) 952.

[4] M.G. Dowsett, A. Adriaens, Nucl. Instr. and Meth. B 226 (2004) 38.

[5] M. Schreiner, M. Grasserbauer, P. March, Fres. Z. Anal. Chem. 331 (1988) 428.

[6] L.R. Riciputi, J.M. Elam, L.M. Anovitz, D.R. Cole, J. Archaeol. Sci. 29 (2002) 1055.

[7] K. Hallett, D. Thickett, D.S. McPhail, R.J. Chater, Appl. Surf. Sci. 203-204 (2003) 789.

[8] N. Lacoudre, T. Beldjoudi, J. Dugot, Metal 98 (1998) 265.

[9] V. Costa, Rev. Conserv. 2 (2001) 18.

[10] M.C. Bernard, E. Douvergne, M. Evesque, M. Keddam, H. Takenouti, Corros. Sci. 47 (3) (2005) 663.

[11] J.L. Maul, S.B. Patel, in: G. Gillen, R. Laureau, J. Bennett, F. Stevie (Eds.), Secondary Ion Mass Spectrometry SIMS XI, John Wiley \& Sons, 1998, p. 707.

[12] M.G. Dowsett, N.S. Smith, R. Bridgeland, D. Richards, A.C. Lovejoy, P. Pedrick, in: A. Benninghoven, B. Hagenhoff, H.W. Werner (Eds.), Ion Mass Spectrometry SIMS X, John Wiley \& Sons, 1997, p. 367.

[13] K. Wittmaack, Phil. Trans. R. Soc. Lond. A354 (1996) 2731.

[14] M. Hirayama, Diss ETH nr. 12333, Zurich, 1997. 